\title{
The Stark Splitting of Millimeter Wave Transitions of Water
}

\author{
Yardley Beers* and Gerald P. Klein* \\ Institute for Basic Standards, National Bureau of Standards, Boulder, Colorado 80302
}

(June 9, 1972)

\begin{abstract}
This paper first discusses the technique of making Stark measurements at millimeter wavelengths. The details of correcting for residual overlap between the lines, the effects of modulation, and of the field inhomogeneity are discussed. Finally the measured frequencies and the empirical Stark coefficients for one $\mathrm{H}_{2} \mathrm{O}$, and one $\mathrm{D}_{2} \mathrm{O}$, and five $\mathrm{HDO}$ lines between 85 and $250 \mathrm{GHz}$ are given. The final analysis of the data to give values of the dipole moment will be given in another paper.
\end{abstract}

Key words: Millimeter waves; Stark effect; water.

\section{Introduction}

This paper contains a partial report upon some Stark measurements made upon millimeter wave transitions of water with the objective of obtaining improved information on the dipole moment. In this work, in obtaining the highest accuracy with the available equipment, it was necessary to apply a number of corrections not employed by previous workers. The bulk of this paper is devoted to a discussion of these corrections and the experimental techniques that were employed. Also contained are values of the measured frequencies and of empirical Stark coefficients for a number of lines.

An extensive analysis is required to obtain values of the dipole moment from the empirical constants. Because water is a very light molecule, there are special problems in carrying out such an analysis. Centrifugal distortion effects result in a significant mixing of rigid rotor states, and the line strengths which are required to carry out the analysis are linear combinations of rigid rotor line strengths. Furthermore there is need for a number of theoretical corrections such as those for the quartic Stark effect and the induced polarizability. Therefore, a discussion of the analysis must be extensive. This analysis and the final results are contained in a second paper [1]. ${ }^{1}$ A preliminary report of a portion of the work has been published elsewhere [2].

\section{Apparatus}

The apparatus used in this work was conventional in design. The absorption cell employed a parallel plate wave guide operating in the TEM mode with the Stark voltage applied between the plates. These were made of brass and were $20 \times 3 \times \frac{1}{2}$ in. Prior to gold plating they were ground flat. The upper plate rested upon four quartz spacers at the corners, and these spacers rest upon the corners of the bottom plate, which is electrically grounded. The signal was fed in and out by horns having aperatures of $5.1 \times$ $0.21 \mathrm{~cm}$ and apex to mouth distances of $8.1 \mathrm{~cm}$. Each was equipped with a plastic lens with a focal length equal to the apex to mouth distance. The horns were attached to RG 138/u (WR8) waveguide with a cutoff at about $73 \mathrm{GHz}$. The horns were located inside the vacuum chamber, but their positions could be changed for alinement purposes by some elaborate mechanisms. Vacuum seals were made by placing thin plastic films in the first waveguide joints. The transmission loss decreased with increased plate separation. The voltage breakdown of the cell was independent of the plate spacing in the range of interest. A spacing of $0.5 \mathrm{~cm}$ seemed to provide an optimum compromise between transmitted signal and maximum obtainable field strength.

The signal was generated by a klystron operating in the region between 40 and $75 \mathrm{GHz}$ and a crossed

*Quantum Electronics Division, National Bureau of Standards, Boulder, Colorado 80302

${ }^{1}$ Figures in brackets indicate the literature references at the end of this paper. 
waveguide crystal frequency multiplier of conventional design. The frequency of the klystron was phase locked to a harmonic of an X-Band klystron, whose frequency was locked to a harmonic of a crystal oscillator. The frequency could be swept by applying motor drive to the shaft of a variable frequency oscillator operating between 5 and $25 \mathrm{MHz}$ which was included in the servo loop of the X-Band klystron. The frequencies of the variable frequency oscillator and the three crystal oscillators in the stabilization circuits were measured with a counter, whose calibration was checked against the NBS Standard.

Both run-in and cartridge crystal detectors were used, but usually the former were preferable with regard to sensistivity. These were of various waveguide sizes, and the lowest harmonics could be rejected by the selection of waveguide size. However, there is some reason to believe that passing lower harmonics may improve the signal-to-noise ratio, but at the expense of difficulties in tuning-up.

With operation at $240 \mathrm{GHz}$ using the fourth harmonic of a $60 \mathrm{GHz}$ klystron, the third harmonic can be peaked up at about five positions of each of the three plungers, giving about 125 combinations. These are not equivalent with respect to the fourth harmonic, and it is necessary to try all of them systematically to find the best one. We had micrometer divisions engraved upon the plungers. These aid in keeping records of which combinations we have tried or in resetting on the optimum one once we have found it. Alternatively, some times we have employed the established technique of using centifugal distortion effects in linear molecules for peaking up a desired harmonic [3]. In such cases, the engraved scales are useful in interpolating the approximate settings between the frequencies where we can optimize the system by this method.

Usually when the detector waveguide was different from the RG 138/u size of the output horn, we butted the detector waveguide directly against the RG 138/u waveguide if the flanges matched. Although a wide assortment of tapered transitions were available, we found we could obtain at least as good a signal, if not a better one, without any in the circuit; apparently the tuning adjustment on the detector mount was able to tune out the impedance mismatch of the waveguide discontinuity. A de bias on the multiplier crystal occasionally improved performance, but one was never found to improve the detector performance.

The dc Stark voltage was obtained from a regulated supply which could be used with either polarity, and in the taking of final data, equal numbers of measurements were made with each polarity. The maximum field that could be used prior to break-down of the cell was $8 \mathrm{KV} / \mathrm{cm}$, and, except for the $7_{35} \leftarrow 6_{42}$ transition of HDO, which has an abnormally large Stark splitting, data were generally taken between 5 and $8 \mathrm{KV} / \mathrm{cm}$. Superimposed upon the dc field was a sinusoidal ac field, variable from zero to $150 \mathrm{~V} / \mathrm{cm}$, which allowed the use of phase sensitive detection. The method for correcting for the effects of the ac field and the need for precise control of its wave form will be discussed later. A wide selection of modulation frequencies was tried, and the optimum seemed to vary between 4 and $20 \mathrm{KHz}$ depending upon circumstances. On the low side it was limited by interference from harmonics of power line frequency, and $4 \mathrm{KHz}$ was the lowest used. To the contrary, many workers, including one of the present authors, have found that frequencies of the order of $100 \mathrm{KHz}$ give optimum sensitivity in the conventional microwave region. However, there the detectors have much greater conversion gains, and the system is much more vulnerable to small shot noise generated in the klystron.

A zero based square wave generator giving voltages up to $1500 \mathrm{~V}$ (field strengths up to $3 \mathrm{KV} / \mathrm{cm}$ ) was available for observing and measuring the frequencies of the zero field lines, but this was not used in Stark measurements. For tuning up the system, no Stark voltage was applied, but the sinusoidal ac generator was applied to the multiplier crystal, and phase sensitive detection was used. This provided a very sensitive method for making initial adjustments of either alinement or of tuning: no matter how badly the system was in adjustment, it was almost always possible to get some detectable signal through it, and then it was easy to maximize the received signal. For the final alinement of the geometry a very strong Stark modulated line of $\mathrm{CH}_{3} \mathrm{CN}$ was peaked up.

The preamplifier which was connected between the crystal detector and the phase sensitive detector or cathode-ray oscilloscope was built for the project and provided some flexibility. A number of different input networks and impedance step-up ratios between the crystal and the grid of the first tube were available by the turning of a switch. Usually an impedance step-up of about 4:1 obtained by a high-fidelity AF transformer, gave best results. This was equipped with an extra external mu metal shield to cut down magnetic pick-up. Originally the amplifier provided the selection between cascode and pentode input stages by turning a switch, but in this application the cascade stage never outperformed the pentode one, and later it was dismantled. Built in to the preamplifier was a narrow band filter consisting of a resonant $\mathrm{L}-\mathrm{R}-\mathrm{C}$ circuit, and this was usually used instead of the narrow band filter contained in the commercially built phase sensitive detector, although there was little difference with regard to sensitivity. At various times this preamplifier has been compared to a number of commercial ones, both vacuum tube and solid state, but none has exceeded it in sensitivity.

The vacuum system employed an oil diffusion pump and a thermocouple gage, whose calibration presumably was correct for air. Stark data were taken at pressures between 1 and $10 \mu \mathrm{m}$ nominal reading on this gage. At these pressures the linewidth is essentially independent of the pressure.

The millimeter wave and Stark fields were nominally parallel, and we should have observed only $\Delta M=0$ transitions. However, we did observe the strongest $\Delta M= \pm 1$ transition of the $3_{13} \leftarrow 2_{20}$ line of water, and we estimated that the $\Delta M= \pm 1$ transitions were 
weaker than the $\Delta M=0$ transitions as a group by a factor 18. For simplicity in the following discussion we shall ignore the presence of the $\Delta M= \pm 1$ transitions whenever possible.

\section{Calibration}

The Stark splitting of any spectral line is a function of the product of the dipole moment and the field strength $E$. Therefore, it is necessary to know the field strength in order to determine the dipole moment. In principle this can be found from the voltage difference between the plates and their spacing. However, there are problems in determining the average spacing because of fringing of the dc and rf fields (which may be different) and because of the lack of flatness and parallelism of the plates. These problems are less severe with a molecular beam electric resonance apparatus than with an absorption apparatus like the present one since the beam can largely be confined to the central portion of the plates where the field is highly uniform. On the other hand, the beam type of apparatus is less flexible because of detector problems and can not be used with all of the spectral lines that can be observed with an absorption apparatus. Accordingly, as many previous workers have done, we determine our average spacing by observing the Stark splitting of the molecule OCS, whose dipole moment has been measured very accurately by the beam method in other laboratories. We define the "calibration constant" as the factor by which field strength values based upon geometrical determinations of the spacing must be multiplied to give OCS Stark splittings that are consistent with its dipole moment.

The calibration constant can be found in the first order by direct observation of the Stark splitting of OCS. For this purpose we used by $J=7 \leftarrow 6$ transition, which was the lowest that could be observed with the RG 138/u waveguide used on our horns. However, we recognized that there was the need for a second order correction because the field inhomogeneities affect the unknown and OCS measurements slightly differently. As a result it is not only necessary to make small corrections to the individual measurements but to modify the value of the calibration constant slightly. A discussion of these matters is postponed to section 7 .

The voltage in our experiment was measured by means of a voltage divider composed of high stability resistors, a commercial potentiometer, and a standard cell. The resistors and cell were checked occasionally by a calibration group at NBS. The voltage divider was also checked against another high quality voltage divider, [4] and also some voltage measurements were checked against a digital voltmeter. The various methods agreed at least within one part in $10^{4}$, and for all practical purposes the voltage measurements could be considered as exact.

The geometrical value of the plate spacing was determined from the lengths of the four quartz spacers at the four corners of the cell. Their lengths were known to a couple parts in $10^{4}$, and they were equal within this accuracy. However, when this value was used with Stark splittings of OCS, the dipole moment was about a percent and a half too large. Then, a ball gage was slipped through a couple of the ports in the wall of the vacuum chamber, and it was used to measure the spacing at the few positions that were accessible. While the measurements were not very precise, they showed definitely that the spacing was smaller at the center than at the corners, and the magnitude was as consistent with the OCS measurements as could be expected.

The most obvious value of the calibration constant is the ratio of the standard value of the dipole moment of OCS to its apparent value using the geometrical value of the spacing. However, we have decided that this was not the best value because some of the higher order corrections are not correct when this is used. Instead, we have assumed a value of the calibration factor and then, after applying all corrections to the data, we ran it through the same computer program as we use for the reduction of the data of the unknown. If the value of the OCS dipole moment differed from the accepted one by more than a couple parts in $10^{4}$, we tried other calibration factors until we found one that does give a value of the OCS dipole moment that lies within this limit. Then, simple interpolation was used to obtain the final factor, which was 1.01511. Some further justification for this elaborate process has resulted from the fact that the standard deviation of it is significantly decreased as the apparent value approached the standard value.

As a standard value of the dipole moment of OCS we have used $0.71521 \pm 0.00020 \mathrm{D}$ due to Muenter [5]. While the work has been in progress, an improved value of $0.71512 \pm 0.00003 \mathrm{D}$ has been published by De Leeuw and Dymanus [6]. These values are in excellent agreement, and the accuracy of both exceeds the precision of the present work. It is to be observed that these standard values were obtained by observation of transitions between $M=0$ and $M \pm 1$ levels of the $J=1$ state, while we employed the $J=7 \leftarrow 6$ transition. If subsequent investigations of centrifugal distortion effects in OCS should reveal a detectable change in the dipole moment, our values will have to be corrected.

In our reduction of data we employed for Planck's constant the value $6.6256 \times 10^{-27} \mathrm{erg} \mathrm{s}$ [7].

In the succeeding sections we shall describe the experimental and theoretical corrections applied to the data and the method of reducing it.

\section{Modulation Correction}

With water and OCS the lowest nonvanishing order of the Stark effect is the second order, and the next is the fourth order. In considering only the second order as an approximation, we may write for the frequency of the $M \leftarrow M$ Stark component of a line of zero field frequency $f_{0}$

$$
\begin{gathered}
f_{M}=f_{0}+K_{M} E^{2} \\
=f_{0}+K_{M}\left(E_{0}+e \cos \omega t+e_{0}\right)^{2},
\end{gathered}
$$


where $K_{M}$ is the Stark coefficient, $E$ is the total applied field, $E_{0}$ is the applied dc field, $e$ is the amplitude of the modulation, assumed to be approximately cosinusoidal with an angular frequency $\omega$, and $e_{0}$ is the dc term in the Fourier analysis of the modulation waveform. The $e_{0}$ term is included because the positive and negative half cycles are not identical in shape.

It is easy to show that the time averaged value of the frequency is

$$
\bar{f}_{M}=f_{0}+K_{M}\left(E_{0}^{2}+\frac{1}{2} e^{2}+2 E_{0} e_{0}+e_{0}^{2}\right) .
$$

The first two terms on the right give the uncorrected value of the frequency. It is valid to assume that $e_{0}$ is small compared to $e$. Therefore the last term may be ignored. However, since $e_{0}$ in the fourth term is multiplied by the large factor $E_{0}$, this term may exceed the third "rectification" term in $e^{2}$ under some circumstances. It is to be supposed that $e_{0}$ is at least roughly proportional to $e$. Therefore, if the fourth term is large compared to the third, the frequency shift is proportional approximately to $e$, while, if the converse is true, the shift is proportional to $e^{2}$.

At any rate, it is desirable to make the shift as small as possible. Obviously the third term cannot be avoided, but the fourth can be avoided or reduced by careful control of the waveform. Here sine wave modulation has a distinct advantage over square wave, since it is easy to design a filter in the form of a resonant parallel $\mathrm{L}-\mathrm{R}-\mathrm{C}$ circuit which can materially improve the waveform.

In general, our method of making the correction experimentally is to measure $\bar{f}_{M}$ as a function of $e$ and extrapolate to zero $e$. The first line investigated was the $4_{22} \leftarrow 4_{23}$ line of HDO, and part of our data on this line were obtained before we became aware that $e_{0}$ might be significant. We found that $\bar{f}_{M}$ had a very nearly linear dependence upon $e$ instead of the square law dependence we had expected. Then we used a filter, and the experimental correction decreased by nearly an order of magnitude. The filter consisted of a parallel resonant circuit connected in parallel with the output of the modulator. It employed a $125 \mathrm{mH}$ choke coil and whatever capacitance was required to produce resonance at the modulation frequency.

With the filter in, the situation is still not completely in accord with theory, as the correction seems to vary from day to day, and at times even appears to reverse in sign. Sometimes a linear fit appears to be better and sometimes a quadratic one does, but by fiat, if nothing else, we have standardized on a quadratic fit. However, we feel that about 100 percent error should be assigned to these corrections. On the other hand, it should be said that the corrections are small (see table 1) and the random error in determining them is probably of the order of 100 percent. Because of the capriciousness of this correction, we have decided that it can not be determined at a time different from the time our main data are taken. Therefore, every time we take data, we take it at three different values of $e$ and extrapolate to zero quadratically. An extrapolation based on three values is not ideal, but it has been necessary to compromise on the basis of expediency.

\section{Overlap Corrections}

Acceptable data were taken only under conditions when the various Stark components ordinarily would be considered as being well resolved. However, with the accuracies we were trying to obtain, it was necessary to make corrections for the fact that each component is superimposed upon the tails of all the others. Approximately, the effect of these tails is to make the baseline appear to be tilted, with the result that the maximum of absorption is displaced slightly. With the type of modulation used, the observed line shape is approximately that of the derivative of the absorption-a so-called "dispersion" - curve, and the zero cross-over corresponds to the maximum of absorption. By assuming that the true line shape is Lorentzian and that the separation is large compared to the linewidth, and by retaining the first two terms of a binomial expansion, it is possible to show that the correction to be added to the frequency $f_{i}$ due to the tail of another component of frequency $f_{j}$ is given by

$$
\delta F_{i}=\frac{a b^{4}}{16\left(f_{i}-f_{j}\right)^{3}}+\frac{3 a b^{8}}{256\left(f_{i}-f_{j}\right)^{7}},
$$

where $b$ is the average full width at half power and $a$ is the ratio of the apparent intensity of the perturbing component to the apparent intensity of the perturbed component.

The factor $a$ is in turn composed of three factors. First, there is the factor $a_{1}$, the true intensity ratio as calculated from quantum theory. Secondly, there is the polarization factor $p$, since both $M \leftarrow M$. and $M \leftarrow M \pm 1$ components were present. When the two components are of the same polarization, $p=1$; when they are opposite, $p=1 / 18$ or 18 , as appropriate. Thirdly, since we employed a modulation method, it was appropriate to include a relative modulation coefficient, which was given by the ratio of the $K_{M}$ 's, taking into account their signs. When the components are on the same side of the zero field line, the sign of the correction is to pull the lines apart, while if they are on opposite sides, the sign of the correction is to bring them together.

It is comforting to note that even in the case when $f_{i}-f_{j}=b$, when the approximation cannot be expected to be very valid, the first term (for $a=1$ ) is only $(1 / 16) b$, and the second is only $(3 / 256) b$.

In practice it is convenient to express the frequency differences in the denominator in terms of the field strength $E$ by use of eq (1). Equation (4) with this modification must be summed over all perturbing components $j$. A computer program was written for carrying out this summation and printing out the two terms separately with $b$ and $E$ given nominal values. Then the correction was found in actual cases by multiplying the terms by appropriate power of the ratios of the actual to nominal values. The second term was mainly useful as an indication of danger: when it 
TABLE 1. Summary of corrections

\begin{tabular}{l|c|c|c|c|c|c}
\hline \hline \multirow{2}{*}{ Molecule } & Line & \multirow{2}{*}{$\begin{array}{c}\text { Linewidth } \\
(\mathrm{MHz})\end{array}$} & \multicolumn{2}{|c|}{ Max. correction per L.W. } & \multirow{2}{*}{$\begin{array}{c}\text { Standard dev. } \\
\text { fit per L.W. }\end{array}$} \\
\cline { 4 - 6 } & & & Modulation & Overlap & $E^{4}$ & \\
\hline & & & & & & \\
OCS & $7 \leftarrow 6$ & 0.16 & $4 \times 10^{-2}$ & $<10^{-2}$ & $2 \times 10^{-2}$ & $6 \times 10^{-5}$ \\
$\mathrm{H}_{2} \mathrm{O}$ & $3_{13} \leftarrow 2_{20}$ & .50 & $5 \times 10^{-2}$ & $4 \times 10^{-3}$ & $6 \times 10^{-6}$ & $2 \times 10^{-3}$ \\
HDO & $7_{35} \leftarrow 6_{42}$ & .40 & $4 \times 10^{-2}$ & $2 \times 10^{-2}$ & $1 \times 10^{-1}$ & $7 \times 10^{-2}$ \\
HDO & $5_{15} \leftarrow 4_{22}$ & .42 & $2 \times 10^{-2}$ & $2 \times 10^{-3}$ & $1 \times 10^{-2}$ & $2 \times 10^{-2}$ \\
HDO & $4_{22} \leftarrow 4_{23}$ & .40 & $3 \times 10^{-1}$ & $3 \times 10^{-3}$ & $5 \times 10^{-3}$ & $5 \times 10^{-2}$ \\
HDO & $7_{34} \leftarrow 7_{35}$ & .37 & $5 \times 10^{-2}$ & $3 \times 10^{-3}$ & $6 \times 10^{-5}$ & $7 \times 10^{-2}$ \\
HDO & $2_{11} \leftarrow 2_{12}$ & .58 & $5 \times 10^{-2}$ & $1 \times 10^{-4}$ & $1 \times 10^{-4}$ & $4 \times 10^{-2}$ \\
D $_{2} \mathrm{O}$ & $4_{14} \leftarrow 3_{21}$ & .40 & $5 \times 10^{-2}$ & $3 \times 10^{-2}$ & $8 \times 10^{-5}$ & $5 \times 10^{-2}$ \\
\hline
\end{tabular}

Values shown are the maximum values applicable to any of the final data. Average values are generally considerably smaller.

The linewidths given above are full widths at half maximum as observed at low field strengths and with small levels of modulation and at low pressures (about $5 \mu \mathrm{m}$ ).

For details of corrections for inhomogeneity of fields, see text.

became large, either no serious data were obtained or the calculation had to be modified as below.

With the $7_{35} \leftarrow 6_{42}$ line a special problem developed. The Stark coefficient $\left(K_{M}\right)$ of the $6_{42}$ level is abnormally large because of the near coincidence with $6_{43}$ level, only $2395 \mathrm{MHz}$ below. Therefore each $M \leftarrow M$ component is flanked by unresolved $M \leftarrow M-1$ and $M \leftarrow M+1$ components, and for these the above approximations do not hold. For this case, with the assumptions that the separation is small compared to $b$ and that the peak of the perturbed component is displaced by only a small fraction of $b$, it can be shown that the correction to be added is

$$
\delta \boldsymbol{F}_{i}=a\left(f_{i}-f_{j}\right) /(1+a) .
$$

In these cases, the appropriate terms were deleted from the summation of eq (4) and then corrections from eq (5) were added. In one or two other special cases when the computer program produced an abnormal value for the second term in eq (4), investigation showed that there was an accidental near coincidence between a $M \leftarrow M$ component and some $M \leftarrow M \pm 1$ component. Then this term was deleted from eq (4) and evaluated by eq (5).

Maximum values of overlap corrections are shown in table 1. On most occasions, the correction was completely negligible. The general validity of eqs (4) and (5) was checked in a couple of cases by actually calculating line shapes.

\section{Higher Order Stark Effects}

It was necessary in the analysis of data, of course, to determine whether the fourth order terms were significant and, if so, to correct for them. We are indebted to $\mathrm{S}$. A. Clough for providing us with a very flexible computer program which allowed us to make least squares fits to several different alternate sets of parameters. It not only gave us the values of the parameters but also the standard deviations in them and the correlation coefficients between the errors. Unsatisfactory results were obtained when this program was used to evaluate the fourth order coefficients empirically, as the correlation coefficients were very large. Then Clough supplied us with fourth order corrections he calculated by use of fourth order perturbation theory, [8] in some cases by summing more than a thousand terms. In the analysis of data, the contributions of the fourth order terms have been subtracted off. In all cases they are small, and in most they are negligible.

Table 1 shows the maximum values of various corrections expressed in fractions of a linewidth. Also shown for comparison are the standard deviations of fit expressed in a similar way. The average values of the corrections were generally much smaller, and except for the modulation correction, the errors in the corrections were small compared to the corrections themselves. Therefore, these corrections should have contributed little error to the final results.

\section{Effect of Field Inhomogeneities}

The rigorous method of dealing with the width of a spectral line that has been broadened by a number of causes, of course, is to determine the distribution function due to each cause and then combine them. Obviously, this approach is impractical in our case. Instead we employ an intuitive formula that has had some acceptance. If $b_{i}$ is the linewidth due to some cause of broadening $i$, then the total linewidth is given by

$$
b=\sqrt{\sum_{i} b_{i}^{2}}
$$

The intuitive justification for this formula is that each cause of broadening may be considered as an independent source of noise with respect to the definition of the line frequency, and sources of noise are normally combined in this fashion.

If $E$ is the $\mathrm{rms}$ variation in the field strength as averaged over the effective volume of the sample, then from eq (1) we may show that the width

$$
b_{1}=2\left(f_{M}-f_{0}\right) \frac{\Delta E}{E} .
$$


Let $b_{0}$ denote the limiting linewidth at low pressures (composite of Doppler and collision broadening) in the absence of the effect of inhomogeneities. Then by use of eqs (6) and (7) and by use of the binomial theorem, for low fields, approximately

$$
b=b_{0}+2\left[\frac{\left(f_{M}-f_{0}\right)}{b_{0}}\left(\frac{\Delta E}{E}\right)\right]^{2} .
$$

By fitting some data to eq (8), it was estimated that $\Delta E / E$ was about 0.005 . This is somewhat less than what we might have expected from the calibration factor of 1.01511. Apparently the plates are fairly flat in the middle, but are thinner at the corners where the spacers are located. When they were ground, they were probably clamped at the corners.

Broadening causes no error in the Stark measurements if the broadening is symmetrical, but this is unlikely. Furthermore, even if the broadening is unsymmetrical, in the first order it should cause no error since it will have affected measurements on both OCS and upon water more or less in the same way. In the second order there may be some error because the two may not have been made under exactly the same conditions. To correct for these errors, we have carried out some line shape studies.

Nominally, we take for the frequency of a Stark component as the cross over frequency (averaged over upward and downward sweeps) on the output of the phase sensitivity detector, whose response is very nearly the derivative of the absorption curve. This corresponds to the peak of the absorption curve, or the most probable frequency, which we shall denote as $f_{c}$. On the other hand, what is significant in the reduction of Stark data is the frequency as averaged over the absorption curve, which we shall denote as $\bar{f}$. Accordingly, we have written a computer program for taking data points that have been transcribed from the recordings of the shapes of Stark components and performing all the necessary integrations and calculating $\bar{f}$.

Without automation, it is not practical to apply this process to all of the individual measurements. Therefore, we evaluated the effect under the most favorable experimental conditions and used these data along with reasonable assumptions to derive corrections to be applied to all the individual measurements. Let us define two quantities $X$ and $Y$ as follows:

and

$$
X=\left(f_{c}-f_{0}\right) / b_{0}
$$

$$
Y=\left(f_{c}-\bar{f}\right) / b_{0} .
$$

It was then assumed that $Y$ is the same function of $X$ for all Stark components, and independent of the molecule. It was now necessary to determine empirically the form of the function.

To determine this relationship, we worked with the $M=4 \leftarrow 4$ component of the $4_{22} \leftarrow 4_{23}$ transition of HDO since this component is well isolated from all others, allowing accurate establishment of a baseline, and since the intensity is favorable.
From the material on the preceding pages, we expected intuitively that $Y$ would be a quadratic function of $X$. We tried least squares fits of the data to several different functions. The fit to a quadratic function was acceptable, but the fit to a linear function was still better. However the best fit was with

$$
Y=0.00181 X^{1.35},
$$

and this was used to correct all the measurements.

In making this correction, there is some difficulty in choosing the value of the linewidth because the linewidths are not the same for all the Stark components of any given transition. The ones with larger Stark coefficients are generally broader. Apparently they are more vulnerable to modulation broadening. Although we used reduced modulation voltages for these components, often it was not practical to reduce them to the point where the modulation broadening became negligible as compared to the inner components.

In applying eq (11) we used the limiting small linewidth for all components. These are the values shown in table 1 . In calculating the overlap corrections we used average values which typically were about twenty-five percent larger.

The maximum value of the normalized frequency shift $X$ in all the data which we used in our analysis is about 45, which corresponds to a correction of 1.4 percent. The average correction, of course, is much smaller. The calibration constant changes from (a) 1.01763, when the corrections are not applied, to (b) 1.01511 , when they are applied. If values of the dipole moment are calculated using the two procedures mentioned above, the largest change is about one part in $10^{3}$. In nearly all cases the standard deviation is about ten percent smaller in case (b) indicating that this procedure is a valid one.

\section{Results}

Data were taken at several different field strengths and on as many of the Stark components as could give significant data. The number of data along with the principal results are listed in table 2 .

The frequencies listed there are those given as one of the fitting parameters given by Clough's computer program. The errors stated are three times the standard deviations given by the program plus an allowance for the uncertainty in the calibration of our counter of 2 parts in $10^{8}$ and 8 parts in $10^{8}$ for servo error.

In a strict sense we never measured the frequency of our millimeter wave oscillator: we only measured the frequencies of the variable frequency oscillator and of the various crystal oscillators in the two servo loops that stabilized the oscillators. Thus, the results are subject to a "servo" error. With a borrowed counter, we measured the frequency of the X-band oscillator directly and compared the values with those computed. We found an agreement within 5 parts in $10^{8}$. We had no means of making a comparable check on the second servo loop, but we assume that it made a comparable error. Thus assuming that they 
TABLE 2. Summary of data

\begin{tabular}{l|c|r|r|r|c}
\hline \hline Molecule & Line & \multicolumn{1}{|c|}{ Meas. freq. $(\mathrm{MHz})$} & $A \mathrm{MHz}$ per $(\mathrm{KV} / \mathrm{cm})^{2}$ & $B \mathrm{MHz}$ per $(\mathrm{KV} / \mathrm{cm})^{2}$ & $\begin{array}{c}\text { Cor. Coef. } \\
A-B\end{array}$ \\
\hline $\mathrm{OCS}$ & $7 \leftarrow 6$ & $83,139.103 \pm .007$ & & & \\
$\mathrm{H}_{2} \mathrm{O}$ & $3_{13} \leftarrow 2_{20}$ & $183,310.094 \pm .013$ & $7.7531 \times 10^{-2} \pm .0034$ & $-4.2180 \times 10^{-2} \pm .0005$ & -0.257 \\
$\mathrm{HDO}$ & $7_{35} \leftarrow 6_{42}$ & $87,962.829 \pm .014$ & $2.771 \times 10^{-1} \pm .067$ & $-4.0463 \times 10^{-1} \pm .0097$ & -0.317 \\
$\mathrm{HDO}$ & $5_{15} \leftarrow 4_{22}$ & $120,778.262 \pm .009$ & $2.2071 \times 10^{-1} \pm .0014$ & $-2.7145 \times 10^{-2} \pm .0008$ & -0.502 \\
$\mathrm{HDO}$ & $4_{22} \leftarrow 4_{23}$ & $143,727.291 \pm .031$ & $-1.1494 \times 10^{-1} \pm .0047$ & $2.6164 \times 10^{-2} \pm .0031$ & -0.807 \\
$\mathrm{HDO}$ & $7_{34} \leftarrow 7_{35}$ & $151,616.218 \pm .019$ & $-9.7694 \times 10^{-2} \pm .076$ & $6.326 \times 10^{-3} \pm .018$ & -0.851 \\
$\mathrm{HDO}$ & $2_{11} \leftarrow 2_{12}$ & $241,561.574 \pm .066$ & $-3.908 \times 10^{-2} \pm .067$ & $1.3629 \times 10^{-1} \pm .0011$ & -0.423 \\
$\mathrm{D}_{2} \mathrm{O}$ & $4_{14} \leftarrow 3_{21}$ & $151,710.418 \pm .014$ & $1.2965 \times 10^{-1} \pm .0028$ & $-4.2556 \times 10^{-2} \pm .0034$ & -0.354 \\
\hline
\end{tabular}

See text for the definitions of the assigned errors.

combine as random errors, we make an allowance of 8 parts in $10^{8}$ for the two together.

In not all cases did we make a measurement at zero field directly, but when such a value was available it was included in the fit. Unfortunately, rarely did the square wave generator give sufficient voltage to give complete resolution, and zero field line appeared to shift with voltage. In such cases we extrapolated to infinite voltage. The directly measured values were in reasonable agreement with those given by the computer program.

Five of these lines have been measured by the Duke group $[9,10]$. Our values on the average are higher than the Duke ones by $33 \mathrm{KHz}$ or 2 parts in $10^{7}$. In view of the fact that Duke results are specified to only the nearest $10 \mathrm{KHz}$, the magnitude of these discrepancies is not very disturbing. What is disturbing is that there is no overlap between these sets of measurements: all of our values are higher than all of the Duke ones. We have corresponded with Dr. De Lucia of the Duke group concerning these systematic differences, but at the present time we are unprepared to give an explanation. Some of these lines have also been measured by Steenbeckliers and Bellet [11]. Their values seem to be intermediate.

In the most general case, according to perturbation theory the Stark coefficient $K_{M}$ (see eq (1)), may be written as

$$
K_{M}=A+B M^{2},
$$

where $A$ and $B$ are both proportional to the square of the dipole moment and where there are functions of line strengths and of the geometry. Table 2 lists values of $A$ and $B$ for the various transitions that we have studied as given by Clough's program after correcting for the fourth order effect but neglecting other higher order effects that might be considered. How to obtain values of the dipole moment and appropriate geometrical parameters from $A$ and $B$ and how to determine the appropriate line strengths to be used requires a lengthy discussion, which, as we have said, we have relegated to another paper [1].
The errors in $A$ and $B$ stated in table 2 are one standard deviation as given by Clough's program. The correlation coefficients between these errors also are listed. In addition to these, it is necessary to include the effects of the error in the value of the dipole moment in OCS and the statistical error in our making the calibration, which together amount to about 4 parts in $10^{4}$. Since $A$ and $B$ are proportional to the square of the dipole moment a perfectly correlated error of 8 parts in $10^{4}$ should be added to those given in the table. Any evaluation of the error in the calculated values of the dipole moment requires a knowledge of these errors and correlation coefficients. In the case of the $3_{13} \leftarrow 2_{20}$ line of water it is possible to calculate a value of the dipole moment with one standard deviation of about 5 parts in $10^{4}$, and this is largely limited by the OCS calibration. The values obtained from the other lines are less accurate and their accuracies are not limited significantly by the errors in the OCS calibration.

The first transition to be studied was the $4_{2 \cdot 2} \leftarrow 4_{23}$ transition of HDO, and these first data were taken without a filter in the modulation circuit, as stated previously. About a year and a half later another set of data were taken with the filter present. The two sets of data gave results that were in good agreement. The numbers given in table 2 are based upon the average of both sets.

In addition to providing us with important computer programs and corrections for the fourth order Stark effect, S. A. Clough has provided a variety of other theoretical advice, and he is playing the leading part in the interpretation of the data [1]. We acknowledge helpful discussions with William S. Benedict on the water spectrum and energy levels. We are indebted to Vernon E. Derr for the suggestion that we undertake this work and to him, R. G. Strauch, and R. E. Cupp for advice on experimental techniques and the loan of some equipment. We also thank Thomas W. Russell for the mechanical design of the absorption cell, 
H. A. Gebbie for his interest in an earlier experiment which helped provide us with the facilities for this work, and M. H. Zamboorie for making some of the first measurements.

\section{References}

[1] Clough, S. A., Beers, Yardley, and Klein, Gerald P., to be published.

[2] Beers, Yardley, Klein, Gerald P., and Clough, S. A., Bull. Am. Phys. Soc. II, 16, 86 (1971).

[3] King, William C., and Gordy, Walter, Phys. Rev. 93, 407 (1954).

[4] Park, J. H., J. Res. Nat. Bur. Stand. (U.S.), 66C (Eng. and Instr.), No. 1, 19-32 (Jan.-Mar. 1962).
[5] Muenter, J. S., J. Chem. Phys. 48, 4544 (1968).

[6] DeLeeuw, F. H., and Dymanus, A., Chem. Phys. Ltrs. 7, 288 (1970).

[7] NBS Miscellaneous Publication 253.

[8] Rothman, L. S., and Clough, S. A., J. Chem. Phys. 55, 504 (1971).

[9] Erlandson, G., and Cox, J., J. Chem. Phys. 25, 778 (1956).

[10] DeLucia, F. C., Cook, R. L., Helminger, P., and Gordy, W., J. Chem. Phys., 55, 5334 (1971).

[11] Steenbeckliers, G., and Bellet, J., Compt. Rendue, 270, 1039 (1970).

(Paper 76A5-740) 